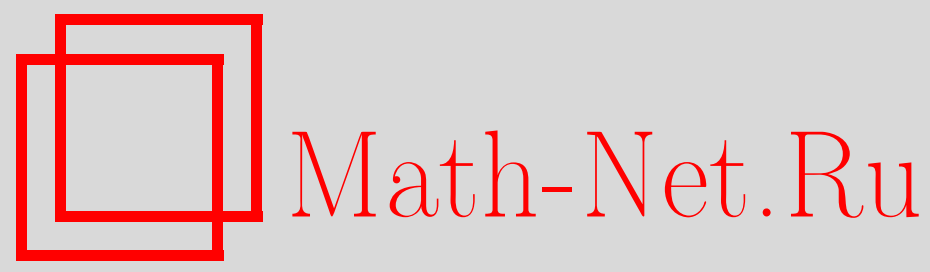

Ю. Г. Рыков, Распространение особенностей типа ударных волн в системе уравнений двумерной газовой динамики без давления, Матем. заметки, 1999, том 66, выпуск 5, 760769

DOI: https://doi.org/10.4213/mzm1219

Использование Общероссийского математического портала Math-Net.Ru подразумевает, что вы прочитали и согласны с пользовательским соглашением http://www.mathnet.ru/rus/agreement

Параметры загрузки:

IP : 3.89 .197 .203

26 апреля 2023 г., $14: 38: 58$

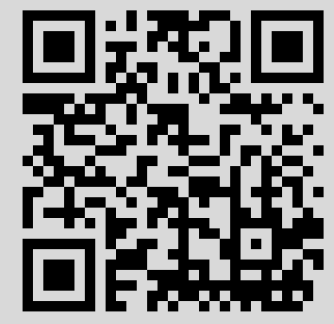




\section{РАСПРОСТРАНЕНИЕ ОСОБЕННОСТЕЙ ТИПА УДАРНЫХ ВОЛН В СИСТЕМЕ УРАВНЕНИЙ ДВУМЕРНОЙ ГАЗОВОЙ ДИНАМИКИ БЕЗ ДАВЛЕНИЯ}

Ю.Г. Рыков

В работе изучается система уравнений, состоящая из двумерного уравнения Бюргерса и уравнения неразрывности. Такая система была предложена Я. Б. Зельдовичем в 1970 году для описания формирования крупномасштабной структуры Вселенной. В настоящей статье для дивергентной формы записи этой системы (являюеейся системой уравнений газовой динамики без давления) сформулировано строгое определение понятия обобщенного решения в терминах мер Радона и получено обобщение соотношений Ренкина-Гюгонио. На основании полученных соотношений показано, что вариационное представление обобщенных решений, справедливое для одномерной системы уравнений газовой динамики без давления, в двумерном случае, вообе говоря, не имеет места.

Библиографоял: 13 названий.

1. Введение. Основные определения. В работе изучаются особенности типа ударных волн для системы уравнений двумерной газовой динамики без давления

$$
\begin{aligned}
& \frac{\partial \rho}{\partial t}+\frac{\partial\left(\rho u_{1}\right)}{\partial x_{1}}+\frac{\partial\left(\rho u_{2}\right)}{\partial x_{2}}=0, \\
& \frac{\partial\left(\rho u_{1}\right)}{\partial t}+\frac{\partial\left(\rho u_{1}^{2}\right)}{\partial x_{1}}+\frac{\partial\left(\rho u_{1} u_{2}\right)}{\partial x_{2}}=0, \\
& \frac{\partial\left(\rho u_{2}\right)}{\partial t}+\frac{\partial\left(\rho u_{1} u_{2}\right)}{\partial x_{1}}+\frac{\partial\left(\rho u_{2}^{2}\right)}{\partial x_{2}}=0, \quad\left(t, x_{1}, x_{2}\right) \in \mathbb{R}_{+} \times \mathbb{R}^{2},
\end{aligned}
$$

где $\left(u_{1}, u_{2}\right)$ имеет физический смысл вектора скорости, $\rho$ имеет физический смысл плотности. Система (1) является нестрого гиперболической системой законов сохранения, имеющей три совпадающих характеристических значения и неполную систему собственных векторов. Благодаря таким свойствам в местах возникновения ударных волн (т.е. местах разрыва вектора скорости) имеет место образование сингулярностей типа $\delta$-функций на поверхности у функции плотности. Поэтому (1) можно рассматривать как

Работа выполнена при финансовой поддержке Российского фонда фундаментальных исследований, грант № 96-01-00377. 
модель процесса концентрации вещества (например, в теории образования крупномасштабной структуры Вселенной, см. ниже). Мы будем исследовать задачу Коши для (1)

$$
\begin{aligned}
\rho\left(0, x_{1}, x_{2}\right) & \equiv 1 \\
u_{1}\left(0, x_{1}, x_{2}\right) & =u_{1}^{(0)}\left(x_{1}, x_{2}\right), \\
u_{2}\left(0, x_{1}, x_{2}\right) & =u_{2}^{(0)}\left(x_{1}, x_{2}\right),
\end{aligned}
$$

где $u_{1}^{(0)}, u_{2}^{(0)}$ являются кусочно $C^{1}\left(\mathbb{R}^{2}\right)$ функциями и будут взяты в специальном виде, чтобы локально сформировать движущийся фронт ударной волны.

Для гладких функций система (1) эквивалентна следующей системе уравнений

$$
\begin{aligned}
& \frac{\partial \rho}{\partial t}+\frac{\partial\left(\rho u_{1}\right)}{\partial x_{1}}+\frac{\partial\left(\rho u_{2}\right)}{\partial x_{2}}=0, \\
& \frac{\partial u_{1}}{\partial t}+u_{1} \frac{\partial u_{1}}{\partial x_{1}}+u_{2} \frac{\partial u_{1}}{\partial x_{2}}=0, \\
& \frac{\partial u_{2}}{\partial t}+u_{1} \frac{\partial u_{2}}{\partial x_{1}}+u_{2} \frac{\partial u_{2}}{\partial x_{2}}=0, \quad\left(t, x_{1}, x_{2}\right) \in \mathbb{R}_{+} \times \mathbb{R}^{2} .
\end{aligned}
$$

Последние два уравнения в системе (3) представляют собой так назьваемое невязкое уравнение Бюргерса, которое было предложено (но в трехмерном случае) Я. Б. Зельдовичем [1] для описания формирования крупномасштабной структуры Вселенной. В дальнейшем этот подход, в частности, рассмотрение всей системы (3), был развит в последующих работах (см., например, [2] и содержащиеся там ссылки) с физической точки зрения. Но поскольку мы фактически имеем дело с законами сохранения массы и импульса, кажется более удобным и отвечающим существу дела как с математической, так и с физической точки зрения использовать систему уравнений в дивергентной форме (1), особенно если имеет место распространение ударных волн. Для одномерного варианта системы (1) частные обобщенные решения были построены в [3] (там также были предложены некоторые численные схемы), а теорема существования обобщенных решений в смысле мер Радона для широкого класса начальных данных была доказана в [4]-[6]. В [4], [5] обобщенные решения были построены с помощью вариационного принципа для подходящего уравнения Гамильтона-Якоби (см. [7]-[10] о вариационном принципе для одного квазилинейного уравнения первого порядка).

Были предприняты определенные усилия, чтобы построить обобшенные решения для (1) по аналогии с одномерным случаем с помощью вариационного принципа, которьй можно написать для двумерного невязкого уравнения Бюргерса и затем обобщить, используя соответствующее уравнение Гамильтона-Якоби. Далеемы покажем, что, вообще говоря, это невозможно. Изучение задач, связанных с многомерными системами законов сохранения, где возникают разные типы особенностей, представляет значительные трудности, см., например, работы [11]-[13]. В настоящей работе исследуется "простейший" вырожденный случай двумерной системы, когда характеристики могут быть вычислены явно. Тем не менее, обобщенные решения системы (1) (см. определение 1 ниже) демонстрируют более сложньй характер поведения по сравнению с обобщенными решениями для одного двумерного уравнения первого порядка типа Гамильтона-Якоби. 
Как уже было отмечено, для гладких функций система (1) эквивалентна системе (3), и при достаточно малых значениях времени и для достаточно гладких начальных данных можно применять метод характеристик. Поэтому до возникновения особенностей решения задачи (1), (2) вьглядят следующим образом

$$
\begin{gathered}
\rho\left(t, x_{1}, x_{2}\right)=\frac{\partial(a, b)}{\partial\left(x_{1}, x_{2}\right)}, \\
u_{1}\left(t, x_{1}, x_{2}\right)=u_{1}(0, a, b) ; \quad u_{2}\left(t, x_{1}, x_{2}\right)=u_{2}(0, a, b),
\end{gathered}
$$

где функции $a\left(t, x_{1}, x_{2}\right), b\left(t, x_{1}, x_{2}\right)$ могут быть найдены из уравнений

$$
x_{1}=a+t u_{1}(0, a, b) ; \quad x_{2}=b+t u_{2}(0, a, b) .
$$

Однако хорошо известно, что характеристики системы (3) могут пересекаться за конечное время даже в случае бесконечно гладких начальных данных. Следовательно, будут образовываться особенности: разрывы для вектора скорости $\left(u_{1}, u_{2}\right)$ и $\delta$-функции для плотности $\rho$, что соответствует процессу конщентрации вещества в некоторых точках или вдоль некоторых кривых. Поэтому необходимо понятие обобщенного решения задачи (1), (2), которое ввиду характера возникающих особенностей естественно формулировать в терминах мер Радона.

ОПРЕДЕЛЕНИЕ 1. Пусть $\left(P_{t}\left(d x_{1}, d x_{2}\right),\left(I_{1}\right)_{t}\left(d x_{1}, d x_{2}\right),\left(I_{2}\right)_{t}\left(d x_{1}, d x_{2}\right)\right)$ - семейства мер Радона, определенных на Борелевских подмножествах $\mathbb{R}^{2}$, слабо непрерывных по $t$ и таких, что $P_{t} \geqslant 0$, a $\left(I_{1}\right)_{t},\left(I_{2}\right)_{t}$ - абсолютно непрерывны относительно $P_{t}$ для почти всех $t>0$. Определим вектор-функцию $\left(u_{1}\left(t, x_{1}, x_{2}\right), u_{2}\left(t, x_{1}, x_{2}\right)\right)$ как производные Радона-Никодима

$$
u_{1}\left(t, x_{1}, x_{2}\right)=\frac{d\left(I_{1}\right)_{t}}{d P_{t}} ; \quad u_{2}\left(t, x_{1}, x_{2}\right)=\frac{d\left(I_{2}\right)_{t}}{d P_{t}} .
$$

Тогда $\left.\left(P_{t},\left(I_{1}\right)_{t},\left(I_{2}\right)_{t}\right)\right)$ назовем обобщенным решением задачи $(1),(2)$, если: 1$)$ для любых функций $f, g, h \in C_{0}^{1}\left(\mathbb{R}^{2}\right)$ (пространство непрерьвно дифференцируемых функций с компактньм носителем) и любых $0<t_{1}<t_{2}<+\infty$ вьполняются интегральные тождества

$$
\begin{gathered}
\iint f\left(x_{1}, x_{2}\right) P_{t_{2}}\left(d x_{1}, d x_{2}\right)-\iint f\left(x_{1}, x_{2}\right) P_{t_{1}}\left(d x_{1}, d x_{2}\right) \\
=\int_{t_{1}}^{t_{2}}\left\{\iint \frac{\partial f}{\partial x_{1}}\left(x_{1}, x_{2}\right)\left(I_{1}\right)_{\tau}\left(d x_{1}, d x_{2}\right)\right. \\
\left.\quad+\iint \frac{\partial f}{\partial x_{2}}\left(x_{1}, x_{2}\right)\left(I_{2}\right)_{\tau}\left(d x_{1}, d x_{2}\right)\right\} d \tau \\
\iint g\left(x_{1}, x_{2}\right)\left(I_{1}\right)_{t_{2}}\left(d x_{1}, d x_{2}\right)-\iint g\left(x_{1}, x_{2}\right)\left(I_{1}\right)_{t_{1}}\left(d x_{1}, d x_{2}\right) \\
=\int_{t_{1}}^{t_{2}}\left\{\iint \frac{\partial g}{\partial x_{1}}\left(x_{1}, x_{2}\right) u_{1}\left(\tau, x_{1}, x_{2}\right)\left(I_{1}\right)_{\tau}\left(d x_{1}, d x_{2}\right)\right. \\
\left.\quad+\iint \frac{\partial g}{\partial x_{2}}\left(x_{1}, x_{2}\right) u_{2}\left(\tau, x_{1}, x_{2}\right)\left(I_{1}\right)_{\tau}\left(d x_{1}, d x_{2}\right)\right\} d \tau
\end{gathered}
$$




$$
\begin{gathered}
\iint h\left(x_{1}, x_{2}\right)\left(I_{2}\right)_{t_{2}}\left(d x_{1}, d x_{2}\right)-\iint h\left(x_{1}, x_{2}\right)\left(I_{2}\right)_{t_{1}}\left(d x_{1}, d x_{2}\right) \\
=\int_{t_{1}}^{t_{2}}\left\{\iint \frac{\partial h}{\partial x_{1}}\left(x_{1}, x_{2}\right) u_{1}\left(\tau, x_{1}, x_{2}\right)\left(I_{2}\right)_{\tau}\left(d x_{1}, d x_{2}\right)\right. \\
\left.\quad+\iint \frac{\partial h}{\partial x_{2}}\left(x_{1}, x_{2}\right) u_{2}\left(\tau, x_{1}, x_{2}\right)\left(I_{2}\right)_{\tau}\left(d x_{1}, d x_{2}\right)\right\} d \tau
\end{gathered}
$$

где знак $\iint$ обозначает интегрирование по всему пространству $\mathbb{R}^{2}$;

2) в слабом смысле при $t \rightarrow+0$

$$
\begin{aligned}
& P_{t} \rightarrow d a d b ; \quad\left(I_{1}\right)_{t} \rightarrow u_{1}(0, a, b) d a d b ; \\
&\left(I_{2}\right)_{t} \rightarrow u_{2}(0, a, b) d a d b .
\end{aligned}
$$

В отношении системы (3) имеет место хорошо известное представление Хопфа-Коула [7] для решений с потенциальной начальной скоростью. Это представление может быть получено с помощь метода малой вязкости (т.е. добавления операторов второго порядка с малым параметром $\varepsilon$ в правую часть последних двух уравнений (3) и последующего устремления $\varepsilon$ к нулю) и позволяет определить местоположение особенностей. А именно,

$$
\left(u_{1}, u_{2}\right)=\nabla_{\left(x_{1}, x_{2}\right)} \Psi\left(t, x_{1}, x_{2}\right)
$$

где

$$
\Psi\left(t, x_{1}, x_{2}\right)=\min _{a, b}\left\{S_{0}(a, b)+\frac{\left(x_{1}-a\right)^{2}}{2 t}+\frac{\left(x_{2}-b\right)^{2}}{2 t}\right\},
$$

$S_{0}$ представляет собой потенциал начальной скорости. Представление (10) имеет место в областях гладкости $\Psi$ и дает возможность найти то множество точек, где возникнут особенности. А именно, сингулярными точками $\Psi$ будут такие точки $\left(t, x_{1}, x_{2}\right)$, что глобальньй минимум по переменным $(a, b)$ выражения в фигурных скобках достигается более, чем в одной точке. Для одномерного случая представление (10) дает возможность построить обобщенное решение для одномерного аналога задачи (1), (2), см. [4], [5] (также и для непостоянной начальной плотности). Оказывается, что в двумерном случае обобщенное решение задачи (1), (2) в смысле определения 1 не может быть построено с помощью представления (10).

В п. 2 будут сформулированы условия на начальные данные (2), которые являются естественньми ограничениями для возникновения ударной волны, и затем, предполагая, что такая ударная волна сушествует и является решением задачи $(1),(2)$ в смысле определения 1, будут выведены формулы для скорости вдоль ударной волны. Эти формулы являются обобщениями соотношений Ренкина-Гюгонио для гиперболических систем законов сохранения. В п. 3 показано, что в случае потенциальной начальной скорости полученные формулы определяют поверхность особенностей (в пространстве $\left.\left(t, x_{1}, x_{2}\right)\right)$, которая, вообще говоря, не совпадает с аналогичной поверхностью, определяемой с помощью представления (10).

Далее ниже буквенные индексы (кроме $i, j$ и $t$ ) будут обозначать соответствующие частные производные. 
2. Распространение фронта ударной волны. Обозначим координаты в плоскости $\mathbb{R}^{2} \times\{t=0\}$ через $(a, b)$. Пусть в плоскости $(a, b)$ существует $C^{1}$ кривая $\mathcal{G}=$ $(A(l), B(l)), l$-параметр вдоль кривой, такая, что $A_{l} \geqslant 0, B_{l} \geqslant 0$ или $A_{l} \geqslant 0, B_{l} \leqslant 0$; $A_{l} B_{l} \neq 0$. Предположим, что кривая $\mathcal{G}$ также может быть задана как решение уравнения $G(a, b)=0, G \in C^{1}\left(\mathbb{R}^{2}\right)$. Тогда рассмотрим следующие начальные данные $(2)$

$$
\begin{aligned}
\rho_{0}(a, b) & \equiv 1, \\
u_{1}^{(0)}(a, b) & =u_{1,-}+\left(u_{1,+}-u_{1,-}\right) H(G(a, b)), \\
u_{2}^{(0)}(a, b) & =u_{2,-}+\left(u_{2,+}-u_{2,-}\right) H(G(a, b)),
\end{aligned}
$$

где $H$ - функция Хевисайда, т.е. $H(\theta)=0$ для $\theta<0, H(\theta)=1$ для $\theta>0$; и выполнены следующие условия:

I) для $(i=1,2)$

$$
u_{i,-}(a, b) \in C^{1}\left(\overline{\mathcal{G}^{-}}\right), \quad u_{i,+}(a, b) \in C^{1}\left(\overline{\mathcal{G}^{+}}\right)
$$

$\mathcal{G}^{-} \equiv\{(a, b): G(a, b)<0\}, \mathcal{G}^{+} \equiv\{(a, b): G(a, b)>0\}$.

II) $A_{l} u_{2,-}-B_{l} u_{1,-} \leqslant 0$ и $A_{l} u_{2,+}-B_{l} u_{1,+} \geqslant 0$ на $\mathcal{G}$ в случае $A_{l} \geqslant 0, B_{l} \geqslant 0$ или $A_{l} u_{2,-}-B_{l} u_{1,-} \geqslant 0$ и $A_{l} u_{2,+}-B_{l} u_{1,+} \leqslant 0$ на $\mathcal{G}$ в случае $A_{l} \geqslant 0, B_{l} \leqslant 0$.

ЗАмЕчАнИЕ 1. 1) Заметим, что условия I) говорят о том, что для всякой точки $\left(a^{*}, b^{*}\right)=\left(A\left(l^{*}\right), B\left(l^{*}\right)\right) \in \mathcal{G}$ существует некоторая область $Q \subset \mathbb{R}^{2} \times\left[0, T\left(l^{*}\right)\right]$, $T\left(l^{*}\right)>0,\left(a^{*}, b^{*}\right) \in Q \cap\{t=0\}$ такая, что преобразования

$$
\begin{aligned}
& x_{1}=a+t u_{1, j}(a, b), \\
& x_{2}=b+t u_{2, j}(a, b) \quad(j=+,-)
\end{aligned}
$$

являются невырожденными для всякого $0<t<T\left(l^{*}\right)$, характеристические прямые $(5)$ с $u_{i}(0, a, b)=u_{i,-}(i=1,2)$ не пересекаются и характеристические прямые $(5) \mathrm{c}$ $u_{i}(0, a, b)=u_{i,+},(i=1,2)$ не пересекаются.

В то же время условия II) говорят о том, что для $0<t<T\left(l^{*}\right)$ существует другая область $Q_{1} \subset Q$, где существуют ровно две характеристические прямые, начинающиеся по разные стороны от кривой $\mathcal{G}$ и приходящие в одну и ту же точку $Q_{1}$ (собственно говоря, поверхность ударной волны должна лежать в множестве $\left.Q_{1}\right)$.

Поэтому приведенные выше условия являются достаточно естественными для того, чтобы предположить существование ударной волны локально по пространству в течение некоторого времени $T\left(l^{*}\right)>0$.

Таким образом, можно искать решение задачи $(1),(11)$ в виде

$$
\begin{aligned}
\rho\left(t, x_{1}, x_{2}\right) & =\rho_{-}+\left(\rho_{+}-\rho_{-}\right) H(S)+\widetilde{P}_{t} \delta(S), \\
\rho u_{1}\left(t, x_{1}, x_{2}\right) & =\rho_{-} u_{1,-}+\left(\rho_{+} u_{1,+}-\rho_{-} u_{1,-}\right) H(S)+\left(\widetilde{I_{1}}\right)_{t} \delta(S), \\
\rho u_{2}\left(t, x_{1}, x_{2}\right) & =\rho_{-} u_{2,-}+\left(\rho_{+} u_{2,+}-\rho_{-} u_{2,-}\right) H(S)+\left(\widetilde{I_{2}}\right)_{t} \delta(S),
\end{aligned}
$$

где $\rho_{j}\left(t, x_{1}, x_{2}\right), u_{i, j}\left(t, x_{1}, x_{2}\right), S\left(t, x_{1}, x_{2}\right) \in C^{1}\left(R_{+} \times R^{2}\right)(i=1,2 ; j=+,-)$ и удовлетворяют (1) в классическом смысле; решение уравнения $S\left(t, x_{1}, x_{2}\right)=0$ представляет 
собой некоторую поверхность $\mathcal{S}$ в $\mathbb{R}_{+} \times \mathbb{R}^{2}$ такую, что $\mathcal{S} \cap\left(\{0\} \times \mathbb{R}^{2}\right)=\mathcal{G} ; \widetilde{P}_{t},\left(\widetilde{I_{1}}\right)_{t},\left(\widetilde{I_{2}}\right)_{t} \in$ $C^{1}(\mathcal{S}) ; H$ - функция Хевисайда, упомянутая выше, $\delta$ - стандартная $\delta$-функция Дирака.

Если обобщенное решение в смысле определения 1 задачи (1), (11) существует, то ввиду формул (6) определен вектор скорости $\widetilde{U}=\left(\widetilde{u}_{1}, \widetilde{u}_{2}\right)$ на поверхности $\mathcal{S}$, и из каждой точки кривой $\mathcal{G}$ можно провести интегральную кривую поля $\widetilde{U}$ на $\mathcal{S}$. Пусть $\left(x_{1}^{s}(t, l)\right.$, $\left.x_{2}^{s}(t, l)\right)$ является соответствующей параметризацией $\mathcal{S}(l$ - параметр вдоль $\mathcal{G})$ и $d\left(x_{1}^{s}\right) / d t=\widetilde{u}_{1}, d\left(x_{2}^{s}\right) / d t=\widetilde{u}_{2}$. Тогда в соответствии с формулами (5), учитьвая условия I), II), определим функции $a_{j}(t, l), b_{j}(t, l) \in C^{1}(\mathcal{S})(j=+,-)$ как координаты начальных точек для характеристик, приходящих в момент времени $t$ в одну и ту же точку поверхности $S$.

Теперь определим отображение

$$
\mathcal{L}:(a, b) \rightarrow\left(x_{1}, x_{2}\right)
$$

следуюшим образом. Вьпустим характеристическую прямую (принимая во внимание (11)) из точки $(a, b)$

$$
x_{1}(\tau)=a+\tau u_{1}^{(0)}(a, b) ; \quad x_{2}(\tau)=a+\tau u_{2}^{(0)}(a, b)
$$

и рассмотрим время $\tau_{0}$ пересечения прямой $(14)$ и поверхности $\mathcal{S}$. Тогда положим $\mathcal{L}_{t}(a, b)=\left(x_{1}(t), x_{2}(t)\right)$, если $\tau_{0}>t$, и $\mathcal{L}_{t}(a, b)=\left(x_{1}^{s}\left(t, l_{0}\right), x_{2}^{s}\left(t, l_{0}\right)\right)$, если $\tau_{0} \leqslant t$, где $l_{0}$ может быть найдено из соотношения $\left(x_{1}\left(\tau_{0}\right), x_{2}\left(\tau_{0}\right)\right)=\left(x_{1}^{s}\left(\tau_{0}, l_{0}\right), x_{2}^{s}\left(\tau_{0}, l_{0}\right)\right)$.

Теперь мы в состоянии сформулировать обобщение соотношений Ренкина-Гюгонио.

Теорема 1. Пусть существует обобщенное в смысле определения 1 решение задачи (1), (11) в форме (12). Тогда справедливы следующие формуль

$$
\begin{aligned}
\widetilde{u}_{1} & =\left({\widetilde{I_{1}}}_{t} / \widetilde{P}_{t}, \quad \widetilde{u}_{2}=\left(\widetilde{I}_{2}\right)_{t} / \widetilde{P}_{t}\right. \\
\widetilde{P}_{t} & =\int_{0}^{t}\left[\left(\left(a_{+}\right)_{\tau}\left(b_{+}\right)_{l}-\left(b_{+}\right)_{\tau}\left(a_{+}\right)_{l}\right)-\left(\left(a_{-}\right)_{\tau}\left(b_{-}\right)_{l}-\left(b_{-}\right)_{\tau}\left(a_{-}\right)_{l}\right)\right] d \tau \\
\left(\widetilde{I}_{1}\right)_{t} & =\int_{0}^{t}\left[u_{1,+}\left(\left(a_{+}\right)_{\tau}\left(b_{+}\right)_{l}-\left(b_{+}\right)_{\tau}\left(a_{+}\right)_{l}\right)-u_{1,-}\left(\left(a_{-}\right)_{\tau}\left(b_{-}\right)_{l}-\left(b_{-}\right)_{\tau}\left(a_{-}\right)_{l}\right)\right] d \tau \\
\left(\widetilde{I}_{2}\right)_{t}= & \int_{0}^{t}\left[u_{2,+}\left(\left(a_{+}\right)_{\tau}\left(b_{+}\right)_{l}-\left(b_{+}\right)_{\tau}\left(a_{+}\right)_{l}\right)\right. \\
& \left.\quad-u_{2,-}\left(\left(a_{-}\right)_{\tau}\left(b_{-}\right)_{l}-\left(b_{-}\right)_{\tau}\left(a_{-}\right)_{l}\right)\right] d \tau .
\end{aligned}
$$

ДокАЗАТЕЛЬСтвО. Пусть на плоскости $(a, b)$ существует некоторое семейство областей $D(\tau)$ с ориентированными гранищами $\partial D(\tau)$ такое, что $D\left(\tau_{1}\right) \subset D\left(\tau_{2}\right)$ для $0<$ $\tau_{1}<\tau_{2}<T$. Пусть $\partial D(\tau)$ является замкнутой кривой $(a(\tau, l), b(\tau, l)), l$ - параметр вдоль кривой, $a, b \in C^{1}\left(\left[\tau_{1}, \tau_{2}\right] \times \mathbb{R}\right)$. Тогда легко проверить справедливость следующей формулы

$$
\frac{d}{d \tau} \iint_{D(\tau)} \varphi d a d b=\iint_{D(\tau)} \frac{\partial \varphi}{\partial \tau} d a d b+\oint_{\partial D(\tau)} \varphi\left(a_{\tau} b_{l}-b_{\tau} a_{l}\right) d l
$$

где $\varphi \in C^{1}\left(\left[\tau_{1}, \tau_{2}\right] \times \overline{\bigcup_{\tau \in\left[\tau_{1}, \tau_{2}\right]} D(\tau)}\right)$. 
Обозначим через П некоторый круг на плоскости $(a, b)$ такой, что $\Pi \times\{t\} \cap \mathcal{S}_{t} \neq \varnothing$, $\mathcal{S}_{t} \equiv \mathcal{S} \cap\{t\} \times \mathbb{R}^{2}$. Обозначим через $D_{-}(t), D_{+}(t)$ области на плоскости $\left(x_{1}, x_{2}\right)$, куда в момент времени $t$ характеристические прямые приходят из точек $\left(a_{-}, b_{-}\right),\left(a_{+}, b_{+}\right)$ соответственно (заметим, что $D_{-}(t) \cup D_{+}(t)=\Pi \times\{t\}$ и $D_{-}(t) \cap D_{+}(t)=\mathcal{S}_{t}$ ). Далее, рассмотрим правую часть интегрального тождества (7). Возьмем $f \in C_{0}^{1}\left(\mathbb{R}^{2}\right)$ такую, что $\operatorname{supp} f \subset$ П. Пусть $D_{j}^{*}(t)=\mathcal{L}_{t}^{-1}\left(D_{j}(t)\right)(j=+,-), S^{*}(t)=\mathcal{L}_{t}^{-1}\left(\mathcal{S}_{t}\right)$. Определим функцию $f_{t}^{*}(a, b)$ так, что $f_{t}^{*}(a, b)=f\left(\mathcal{L}_{t}(a, b)\right)$. Тогда, используя формулу $(17)$, будем иметь

$$
\begin{aligned}
\sum_{j=+,-} & \int_{t_{1}}^{t_{2}} d t\left\{\iint_{D_{j}(t)}\left(f_{x_{1}} u_{1}+f_{x_{2}} u_{2}\right) P_{t}\left(d x_{1}, d x_{2}\right)\right\} \\
= & \sum_{j=+,-} \int_{t_{1}}^{t_{2}} d t\left\{\int \int _ { D _ { j } ^ { * } ( t ) } \left[f_{x_{1}}\left(a+t u_{1}^{(0)}, b+t u_{2}^{(0)}\right) u_{1}^{(0)}(a, b)\right.\right. \\
& \left.\left.+f_{x_{2}}\left(a+t u_{1}^{(0)}, b+t u_{2}^{(0)}\right) u_{2}^{(0)}(a, b)\right] d a d b\right\} \\
= & \sum_{j=+,-} \int_{t_{1}}^{t_{2}} d t\left\{\frac{d}{d t} \iint_{D_{j}^{*}(t)} f_{t}^{*} d a d b\right\} \\
& -\int_{t_{1}}^{t_{2}} d t \int_{S_{t}} f\left(x_{1}^{s}(t, l), x_{2}^{s}(t, l)\right)\left[\left(\frac{\partial\left(a_{-}\right)}{\partial t} \frac{\partial\left(b_{-}\right)}{\partial l}-\frac{\partial\left(b_{-}\right)}{\partial t} \frac{\partial(a-)}{\partial l}\right)\right. \\
& \left.-\left(\frac{\partial\left(a_{+}\right)}{\partial t} \frac{\partial\left(b_{+}\right)}{\partial l}-\frac{\partial\left(b_{+}\right)}{\partial t} \frac{\partial\left(a_{+}\right)}{\partial l}\right)\right] d l .
\end{aligned}
$$

Рассматривая таким же образом правые части интегральных тождеств (8), (9) и принимая во внимание формулы (6), получим доказательство теоремы 1.

Теорема 2. Для любого $l u 0<t<T(l)$ справедливы следующие формуль

$$
\begin{aligned}
& \int_{0}^{t}\left[x_{1}^{s}(t, l)-a_{+}(\tau, l)-t u_{1,+}(\tau, l)\right]\left(\left(a_{+}\right)_{\tau}\left(b_{+}\right)_{l}-\left(b_{+}\right)_{\tau}\left(a_{+}\right)_{l}\right) d \tau \\
& \quad=\int_{0}^{t}\left[x_{1}^{s}(t, l)-a_{-}(\tau, l)-t u_{1,-}(\tau, l)\right]\left(\left(a_{-}\right)_{\tau}\left(b_{-}\right)_{l}-\left(b_{-}\right)_{\tau}\left(a_{-}\right)_{l}\right) d \tau \\
& \int_{0}^{t}\left[x_{2}^{s}(t, l)-b_{+}(\tau, l)-t u_{2,+}(\tau, l)\right]\left(\left(a_{+}\right)_{\tau}\left(b_{+}\right)_{l}-\left(b_{+}\right)_{\tau}\left(a_{+}\right)_{l}\right) d \tau \\
& \quad=\int_{0}^{t}\left[x_{2}^{s}(t, l)-b_{-}(\tau, l)-t u_{2,-}(\tau, l)\right]\left(\left(a_{-}\right)_{\tau}\left(b_{-}\right)_{l}-\left(b_{-}\right)_{\tau}\left(a_{-}\right)_{l}\right) d \tau
\end{aligned}
$$

ДокАЗАТЕЛЬСтво. Рассмотрим формулы (15) и запишем их в следующей форме

$$
\left(x_{1}^{s}\right)_{\tau} P_{t=\tau}=\left(I_{1}\right)_{t=\tau}, \quad\left(x_{2}^{s}\right)_{\tau} P_{t=\tau}=\left(I_{2}\right)_{t=\tau}
$$

Проинтегрируем полученные равенства по $\tau$ от 0 до $t$ и затем, интегрируя по частям, 
получим

$$
\begin{aligned}
& \int_{0}^{t}\left(x_{1}^{s}(t)-x_{1}^{s}(\tau)\right)\left[\left(\left(a_{+}\right)_{\tau}\left(b_{+}\right)_{l}-\left(b_{+}\right)_{\tau}\left(a_{+}\right)_{l}\right)-\left(\left(a_{-}\right)_{\tau}\left(b_{-}\right)_{l}-\left(b_{-}\right)_{\tau}\left(a_{-}\right)_{l}\right)\right] d \tau \\
& \quad=\int_{0}^{t}(t-\tau)\left[u_{1,+}\left(\left(a_{+}\right)_{\tau}\left(b_{+}\right)_{l}-\left(b_{+}\right)_{\tau}\left(a_{+}\right)_{l}\right)-u_{1,-}\left(\left(a_{-}\right)_{\tau}\left(b_{-}\right)_{l}-\left(b_{-}\right)_{\tau}\left(a_{-}\right)_{l}\right)\right] d \tau \\
& \int_{0}^{t}\left(x_{2}^{s}(t)-x_{2}^{s}(\tau)\right)\left[\left(\left(a_{+}\right)_{\tau}\left(b_{+}\right)_{l}-\left(b_{+}\right)_{\tau}\left(a_{+}\right)_{l}\right)-\left(\left(a_{-}\right)_{\tau}\left(b_{-}\right)_{l}-\left(b_{-}\right)_{\tau}\left(a_{-}\right)_{l}\right)\right] d \tau \\
& =\int_{0}^{t}(t-\tau)\left[u_{2,+}\left(\left(a_{+}\right)_{\tau}\left(b_{+}\right)_{l}-\left(b_{+}\right)_{\tau}\left(a_{+}\right)_{l}\right)-u_{2,-}\left(\left(a_{-}\right)_{\tau}\left(b_{-}\right)_{l}-\left(b_{-}\right)_{\tau}\left(a_{-}\right)_{l}\right)\right] d \tau
\end{aligned}
$$

Далее, используя формулы (14), приходим к утверждению теоремы 2.

ЗАмЕчАниЕ 2. Заметим, что выражения (18) подобны формулам, выражающим динамику прилипания в [4], [5]. То есть “частицы”, соответствующие характеристическим прямым, вьпушенным из точек $\left(a_{j}(\tau, l), b_{j}(\tau, l)\right), j=+,-, \tau<t, l$ фиксировано, "сконщентрируются" в одной точке к моменту времени $t$.

3. Сравнение с вариационным представлением. Теперь сравним формулы (16) для ударных волн с формулами для поверхности особенностей, которые могут быть получены из вариационного представления (10) в случае потенциальных начальных данных. Пусть $\mathcal{F}=\left(x_{1}^{v}(t, l), x_{2}^{v}(t, l)\right)$ представляет собой поверхность особенностей, полученную из (10). Тогда $\mathcal{F}$ может быть найдена как решение уравнения

$$
F\left(a_{+}, b_{+} ; t, x_{1}, x_{2}\right)-F\left(a_{-}, b_{-} ; t, x_{1}, x_{2}\right)=0,
$$

где

$$
F \equiv S_{0}(a, b)+\frac{\left(x_{1}-a\right)^{2}}{2 t}+\frac{\left(x_{2}-b\right)^{2}}{2 t},
$$

и $a_{j}\left(t, x_{1}, x_{2}\right), b_{j}\left(t, x_{1}, x_{2}\right)(j=+,-)$ определяются из системы уравнений

$$
F_{a}\left(a, b ; t, x_{1}, x_{2}\right)=0 ; \quad F_{b}\left(a, b ; t, x_{1}, x_{2}\right)=0 .
$$

ТЕОРЕМА 3. Пусть вектор начальной скорости в (11) является потенциальным и потенциал $S_{0}(a, b)$ имеет вид

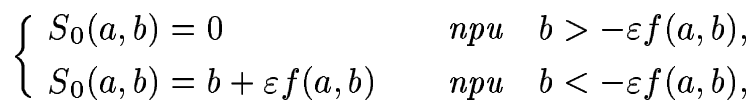

әде $\varepsilon>0$ достаточно мало, а функиия $f \in C^{\infty}\left(\mathbb{R}^{2}\right), f(0,0)=0$, удовлетворяет условиям: существует такая окрестность точки $(a=0, b=0)$, что $f_{a а}(a, 0) \not \equiv$ 0 и кривая, определяемая из уравнения $b+\varepsilon f(a, b)=0$, монотонна для всякого достаточно малого $\varepsilon>0$.

Тогда формуль (15) и (19) задают различные поверхности. 
ДокАЗАТЕЛЬСТво. Из (19) легко вывести, что при достаточно малом $\varepsilon>0$ в окрестности точки $\left(t=0, x_{1}=0, x_{2}=0\right)$ уравнение поверхности особенностей имеет вид

$$
x_{2}=\frac{t}{2}-\varepsilon f\left(x_{1},-\frac{t}{2}\right)+o(\varepsilon) .
$$

Теперь попытаемся найти поверхность особенностей, исходя из формул (15). При $\varepsilon=0$ решение системы (15) вьглядит следующим образом

$$
x_{1}(t, l)=a_{-}(t, l)=a_{+}(t, l)=l, \quad x_{2}(t, l)=b_{-}(t, l)=-b_{+}(t, l)=\frac{t}{2} .
$$

При $\varepsilon>0$ будем искать решение $(15)$ в форме

$$
x_{1}(t, l)=l+\varepsilon \widetilde{x}_{1}(t, l)+o(\varepsilon), \quad x_{2}(t, l)=\frac{t}{2}+\varepsilon \widetilde{x}_{2}(t, l)+o(\varepsilon) .
$$

Тогда, учитьвая соотношения

$$
\begin{aligned}
& x_{1}(t, l)=a_{-}(t, l)=a_{+}(t, l)+t \varepsilon f_{a}\left(a_{+}, b_{+}\right), \\
& x_{2}(t, l)=b_{-}(t, l)=b_{+}(t, l)+t\left(1+\varepsilon f_{b}\left(a_{+}, b_{+}\right)\right),
\end{aligned}
$$

определим $\widetilde{P}_{t},\left(\widetilde{I_{1}}\right)_{t},\left(\widetilde{I_{2}}\right)_{t}$ и найдем

$$
\begin{gathered}
\frac{\partial \widetilde{x}_{1}(t, l)}{\partial t}=\frac{1}{2 t} \int_{0}^{t} f_{a} d \tau \\
\widetilde{x}_{2}(t, l)=-f(l, 0)-\frac{1}{4} \int_{0}^{t} \tau f_{a a} d \tau+\frac{1}{2} \int_{0}^{t} f_{b} d \tau+\frac{1}{4 t} \int_{0}^{t} \tau^{2} f_{a a} d \tau
\end{gathered}
$$

где значения производных функции $f(a, b)$ взяты в точке $(l,-\tau / 2)$. Но функции $(22)$ должны удовлетворять уравнению (21), что, учитьвая (22), эквивалентно выполнению следующего тождества

$$
f\left(l,-\frac{t}{2}\right)-f(l, 0)-\frac{1}{4} \int_{0}^{t}\left(\tau-\frac{\tau^{2}}{t}\right) f_{a a} d \tau+\frac{1}{2} \int_{0}^{t} f_{b} d \tau \equiv 0,
$$

где значения производных функции $f(a, b)$ взяты в точке $(l,-\tau / 2)$. Отсюда, умножая на $t$, затем трижды дифференцируя по $t$ и подставляя $t=0$, получим $f_{a a}(l, 0) \equiv 0$, что противоречит условиям на функцию $f(a, b)$.

ЗАмЕчАниЕ 3. Единственным нетривиальньм примером, известным автору, где вариационное представление обобщенного решения имеет место, является классическая двумерная задача Римана для (1) с постоянной в каждом квадранте $\mathbb{R}^{2}$ начальной скоростью (однако скорость должна иметь сжимающий характер, чтобы не возникало волн разрежения). Но тогда возникает другой тип сингулярности: $\delta$-функция в точке для плотности.

ЗАМЕЧАнИЕ 4. Постоянная начальная плотность выбрана для простоты. После соответствующей модификации формул (16) все рассмотрения остаются справедливыми для непостоянной, достаточно гладкой функции начальной плотности $\rho\left(0, x_{1}, x_{2}\right)$.

Благодарности. Автор выражает благодарность Я.Г. Синаю и В.И. Оселедну за стимулирующие дискуссии, Институту Миттаг-Леффлера, г. Стокгольм и Мюнхенскому университету, факультету теоретической физики, г. Мюнхен, где была написана эта работа. 


\section{СПИСОК ЦИТИРОВАННОЙ ЛИТЕРАТУРЫ}

[1] Zeldovich Ya. B. Gravitational instability: an approximate theory for large density perturbations // Astron. Astrophys. 1970. V. 5. P. 84-89.

[2] Shandarin S. F., Zeldovich Ya. B. The large-scale structure of the Universe: turbulence, intermittency, structures in a self-gravitating medium // Rev. Modern Phys. 1989. V. 61. № 2. P. $185-220$.

[3] Bouchut F. On zero-pressure gas dynamics // Advances in Kinetic Theory and Computing. Series on Advances in Mathematics and Applied Sciences. World Scientific. 1994. V. 22. P. 171-190.

[4] Вейнан И., Рыков Ю. Г., Синай Я. Г. Вариационный принцип Лакса-Олейник для некоторых одномерных систем квазилинейных уравнений // УМН. 1995. Т. 50. № 1. С. 193-194.

[5] Weinan E., Rykov Yu. G., Sinai Ya. G. Generalized variational principles, global weak solutions and behavior with random initial data for systems of conservation laws arising in adhesion particle dynamics // Comm. Math. Phys. 1996. V. 177. P. 349-380.

[6] Grenier E. Existence globale pour le système des gas sans pression // C. R. Acad. Sci. Paris. Sér. 1. 1995. V. 321. P. 171-174.

[7] Hopf E. The partial differential equation $u_{t}+u u_{x}=\mu u_{x x} / /$ Comm. Pure Appl. Math. 1950. V. 3. № 3. P. 201-230.

[8] Lax P. D. Weak solutions of nonlinear hyperbolic equations and their numerical computation // Comm. Pure Appl. Math. 1954. V. 7. № 1. P. 159-193.

[9] Lax P. D. Hyperbolic systems of conservation laws // Comm. Pure Appl. Math.. V. 10. № 4. P. 537-566.

[10] Олейник О. А. Задача Коши для нелинейных дифференциальных уравнений первого порядка с разрьвными начальными условиями // Тр. ММО. 1956. Т. 5. С. 433-454.

[11] Majda A. Compressible fluid flow and systems of conservation laws in several space variables // Appl. Math. Sci. 1984. V. 53. P. 1-159.

[12] DiPerna R. J. Compensated compactness and general systems of conservation laws // Trans. Amer. Math. Soc. 1985. V. 292. № 2. P. 383-420.

[13] Zhang T., Zheng Y.X. Conjecture on structure of solutions of Riemann problem for 2-D gas dynamic systems // SIAM J. Math. Anal. 1990. V. 21. № 3. P. 593-630.

Институт прикладной математики им. М.В. Келдьшша РАН

Поступило

E-mail : rykov@spp.keldysh.ru

22.04 .1998 